Instituto Internacional de Investigación y Desarrollo Tecnológico Educativo INDTEC, C.A.

DOI: https://doi.org/10.29394/Scientific.issn.2542-2987.2021.6.20.11.206-223

OAl-PMH: http://www.indteca.com/ojs/index.php/Revista Scientific/oai

Artículo Original / Original Article

\title{
Blockchain un nuevo concepto de Responsabilidad Social Corporativa en exportadoras de camarón de Ecuador
}

\author{
Autoras: Sabi Samanta Martínez Prado \\ Universidad Técnica de Machala, UTMACH \\ ssmartinez est@utmachala.edu.ec \\ Machala, Ecuador \\ https://orcid.org/0000-0002-3082-6335 \\ Verónica Karina Lascano Campoverde \\ Universidad Técnica de Machala, UTMACH \\ vlascano est@utmachala.edu.ec \\ Machala, Ecuador \\ https://orcid.org/0000-0002-9791-4516 \\ Sandra Sayonara Solórzano Solórzano \\ Universidad Técnica de Machala, UTMACH \\ ssolorzano@utmachala.edu.ec \\ Machala, Ecuador \\ http://orcid.org/0000-0001-6294-7396
}

\section{Resumen}

La responsabilidad social corporativa es el antecedente conocido y comúnmente practicado de las grandes empresas, pero constituye un factor de descuido y marginación de las pequeñas y medianas empresas, por lo que esta es una tarea pendiente por tan importante sector de la economía nacional. En Ecuador, el sector camaronero es uno de los principales generadores de divisas, siendo importante dar a conocer su trazabilidad, procesos productivos, logísticos, ambientales, donde la tecnología blockchain sirve para transparentar estas actividades y ponerlas a conocimiento de clientes y consumidores, garantizando que el producto tiene buenas prácticas productivas y ambientales. El estudio tiene como objetivo describir la tecnología blockchain como un nuevo concepto de Responsabilidad Social Corporativa en exportadoras de camarón en la Provincia de El Oro, Ecuador. Para la recopilación de la información se utilizó el método descriptivo de tipo cualitativo, el análisis documental y la aplicación de la técnica bibliográfica para hacer un acercamiento a información confiable y verídica para su análisis e interpretación. El resultado obtenido es demostrar los beneficios del blockchain en la industria camaronera que necesita optimizar sus procesos de responsabilidad social corporativa en beneficio de sus consumidores y clientes.

Palabras clave: responsabilidad social corporativa; medio ambiente; blockchain; sector camaronero; trazabilidad; empresa.

Código de clasificación internacional: 5304.04 - Comercio exterior.

Cómo citar este artículo:

Martínez, S., Lascano, V., \& Solórzano, S. (2021). Blockchain un nuevo concepto de Responsabilidad Social Corporativa en exportadoras de camarón de Ecuador. Revista Scientific, 6(20), 206-223, e-ISSN: 2542-2987. Recuperado de: https://doi.org/10.29394/Scientific.issn.25422987.2021.6.20.11.206-223

Fecha de Recepción: 09-01-2021
Fecha de Aceptación: 31-03-2021
Fecha de Publicación: 05-05-2021 
OAl-PMH: http://www.indteca.com/ojs/index.php/Revista Scientific/oai

Artículo Original / Original Article

\title{
Blockchain a new concept of Corporate Social Responsibility in shrimp exporters of Ecuador
}

\begin{abstract}
Corporate social responsibility is the known and commonly practiced antecedent of large companies, but it constitutes a factor of neglect and marginalization of small and medium-sized companies, which is why this is a pending task for such an important sector of the national economy. In Ecuador, the shrimp sector is one of the main generators of foreign exchange, being important to publicize its traceability, production, logistics, and environmental processes, where blockchain technology serves to make these activities transparent and make them known to customers and consumers, guaranteeing that the product has good production and environmental practices. The study aims to describe blockchain technology as a new concept of Corporate Social Responsibility in shrimp exporters in the Province of El Oro, Ecuador. For the compilation of the information, the descriptive method of a qualitative type was used, the documentary analysis and the application of the bibliographic technique to make an approach to reliable and truthful information for its analysis and interpretation. The result obtained is to demonstrate the benefits of blockchain in the shrimp industry that needs to optimize its corporate social responsibility processes for the benefit of its consumers and clients.
\end{abstract}

Keywords: corporate social responsibility; environment; blockchain; shrimp sector; traceability; business.

International classification code: 5304.04 - Foreign trade.

\section{How to cite this article:}

Martínez, S., Lascano, V., \& Solórzano, S. (2021). Blockchain a new concept of Corporate Social

Responsibility in shrimp exporters of Ecuador. Revista Scientific, 6(20), 206-223, e-ISSN: 2542-

2987. Recovered from: https://doi.org/10.29394/Scientific.issn.2542-2987.2021.6.20.11.206-223

Date Received:

09-01-2021
Date Acceptance:

31-03-2021
Date Publication: 05-05-2021 


\section{Introducción}

En la sociedad actual, las empresas multinacionales tienen más poder y riqueza que algunos países. Por tanto, la empresa tiene el poder de hacer cambios dentro de la sociedad. Después de 60 años de popularización, la Responsabilidad Social Empresarial (RSE) se ha convertido en una forma de compromiso corporativo. Ahora, la empresa debe considerar el cuidado económico, legal, ambiental y social de toda la comunidad. Los consumidores ahora esperan que las empresas multinacionales, nacionales y locales participen con un mejor rol social, sin embargo, la mayoría de las compañías no han logrado sus objetivos de RSE.

La Responsabilidad Social Corporativa debe estar vinculada a las principales actividades de la empresa para mantener e involucrar los compromisos de la alta dirección en derechos humanos, mejores prácticas laborales, protección de la salud, medio ambiente. Las empresas conocen que es importante combatir el fraude y la corrupción, y tener en cuenta los intereses de los consumidores.

Las redes blockchain se convierten en un elemento tecnológico para comprobar que los objetivos de responsabilidad social empresarial se están cumpliendo, al contener información de sus procesos productivos, los proveedores, insumos utilizados. Mejorar la rendición de cuentas transformará el entorno capitalista actual en un sistema transparente que hará que las empresas multinacionales cumplan con su rol social.

Entre otras cosas, blockchain también proporciona una trazabilidad infalible de registros y notas desde el origen hasta el destino o el consumidor final. Por tanto, la correcta aplicación de esta característica en RSE puede asegurar a los consumidores que los productos que compran no se derivan de actividades ilícitas, como el contrabando, enriquecimiento indebido. Convirtiéndose en una poderosa base de datos, puede combinar cualquier información, como horas de trabajo y otra información tangible. 
Cualquier persona, empresa o consumidor tiene acceso a la información y estar seguro de su autenticidad. Indica Ganne (2018): que los datos generados durante la trazabilidad del producto están disponibles para los participantes de la red, información que está en constante actualización, donde las cadenas de bloques garantizan transparencia que agilizan las transacciones comerciales. Proceso que genera una imagen de ser una organización confiable, seria y comprometida con el consumidor.

La Organización de las Naciones Unidas para la Alimentación y la Agricultura (FAO, 2018): considera que el blockchain se convierte en una oportunidad para la pesquería sostenible, que podrán suministrar información a sus consumidores sobre el proceso de cosecha, insumos utilizados, empaque, transporte y logística, garantizando la trazabilidad del producto, garantizando su calidad.

El blockchain cuyo anglicismo significa cadena de bloques que hace referencia a un protocolo informático que soporta criptomonedas del Bitcoin, para López (2019): se diferencia de esta última que suele operar al margen de monedas fiduciarias, porque el blockchain es un protocolo transparente y confiable.

Con lo que se logra transparentar la cadena logística para que el consumidor conozca la procedencia y procesos productivos involucrados en el producto. El estudio tiene como objetivo describir la tecnología blockchain como un nuevo concepto de Responsabilidad Social Corporativa en exportadoras de camarón en la Provincia de El Oro, Ecuador.

El trabajo empleó el método descriptivo de tipo cualitativo, a lo que se suma el análisis documental para lo que se utilizó información bibliográfica de artículos científicos, páginas web de instituciones gubernamentales y de organizaciones del sector camaronero. Como resultado se pretende demostrar los beneficios del blockchain en la industria camaronera, que es uno de los principales motores de la economía ecuatoriana que necesita optimizar sus 
procesos de responsabilidad social corporativa en beneficio de sus consumidores y clientes.

Mencionando a Esparza, Crespo, González y Fernández (2020): la ventaja de blockchain es que se convierte en un documento, tipo contable, que es compartido y permanece inmutable lo que facilita el proceso en el registro transaccional al que se le puede dar un seguimiento de los activos dentro de una red empresarial. Prácticamente cualquier producto o servicio que contiene valor puede ser rastreado y comercializado dentro de una blockchain, reduciendo los riesgos y costos que involucran toda transacción comercial.

Parafraseando a Machado, Guayasamín y Montoya (2020): el blockchain es la herramienta ideal para las empresas que están haciendo el cambio hacia la Responsabilidad Social Corporativa (RSC) y un argumento de marketing líder para los consumidores-clientes que son cada vez más sensibles y exigentes con respecto al compromiso corporativo, la transparencia y la probidad de gobierno.

Aunque técnicamente accesible, para Benito (2019): el nivel global en el tema sigue siendo muy bajo, y más aún cuando se trata de RSC programable. Además, es más sencillo diseñar un contrato social inteligente al inicio de un nuevo negocio porque, por naturaleza, es más fácil de implementar. Por lo tanto, la industria de activos digitales y el conjunto de startups que la componen, están en primera línea para desarrollarlos e implementarlos.

\section{Metodología (Material y método)}

La investigación, para recopilar la información, usó el método descriptivo y cualitativo para conocer los beneficios del blockchain como mecanismos para mejorar la responsabilidad social empresarial de las empresas camaroneras.

Se utilizó el análisis documental a través de la aplicación de la técnica 
bibliográfica para recabar información de fuentes fiables y confiables como artículos científicos, páginas web de organizaciones privadas e instituciones públicas, para poder hacer un análisis e interpretación de los datos obtenidos procedentes de información estadística de la producción camaronera y los mercados de destino, realizándose figuras y tablas para una mejor interpretación de la investigación.

\section{Resultados (análisis e interpretación de los resultados)}

\subsection{Sector camaronero ecuatoriano}

En una investigación realizada por Zabala y Sánchez (2019): indican que a nivel mundial India es el principal exportador de camarón con una participación del $22.4 \%$ del mercado, le sigue Ecuador con el $15.5 \%$, Vietnam con el $10.3 \%$, indonesia con el $8 \%$, Argentina con el $6.8 \%$.

En este aspecto, Dávila-López, Carvajal-Romero y Vite-Cevallos (2019): comenta que en Ecuador se cultivan dos especies de camarón: el blanco que también se lo conoce como Litopenaeus vannamei que es la que se encuentra en el $95 \%$ de las piscinas camaroneras, por ser una variedad que se ajusta a las características geográficas de nuestro litoral. El otro es el Penaeus stylirostris con una producción del $5 \%$, de menor resistencia al cultivo intensivo.

En ese marco, Vega, Apolo y Sotomayor (2019): señalan que, de las 210 mil hectáreas existentes para la producción de camarón, el 60\% están en Guayas, $15 \%$ en El Oro, 9\% en Esmeraldas, otro 9\% en Manabí, el 7\% en Santa Elena. Convirtiéndose la provincia de El Oro en la segunda de importancia en nuestro país, contribuyendo a su desarrollo económico y social.

En la tabla 1 se expone la producción de camarón de las provincias costaneras del Ecuador, donde la provincia de El Oro le corresponde el $37.68 \%$ de participación, observándose su importancia dentro de este importante sector productivo que contribuye al desarrollo empresarial, social y 
económico del país.

Tabla 1. Producción y participación de camarón según provincias.

\begin{tabular}{|c|c|c|}
\hline Provincia & Producción en millones US\$ & Porcentaje \\
\hline El Oro & 147.977 & 37,68 \\
\hline Esmeraldas & 3.113 & 0,79 \\
\hline Guayas & 169.124 & 43,06 \\
\hline Manabí & 56.067 & 14,28 \\
\hline Galápagos & 97 & 0,02 \\
\hline Santa Elena & 16.379 & 4,17 \\
\hline TOTAL & $\mathbf{3 9 2 . 7 5 7}$ & $\mathbf{1 0 0 , 0 0}$ \\
\hline
\end{tabular}

Fuente: Varela-Véliz, Elizalde, Solórzano y Varela-Véliz (2017).

En un informe realizado por Cavagnaro (2018): sobre el ranking de las 500 empresas más grandes del Ecuador en el año 2017, aparecen dos empresas exportadoras de camarón: Promaoro, S.A. con ventas de 77.24 millones de dólares en el puesto 211; Oceanproduct Cia. Ltda. con 56.59 millones de dólares que lo ubican en el puesto 305; Marecuador Cia. Ltda. tuvo ingresos por 20.84 millones de dólares, pero no se encuentra en el ranking. El producto que envían al exterior es el camarón del tipo Litopenaeus vannamei.

Gráfico 1. Exportaciones de camarón ecuatoriano.

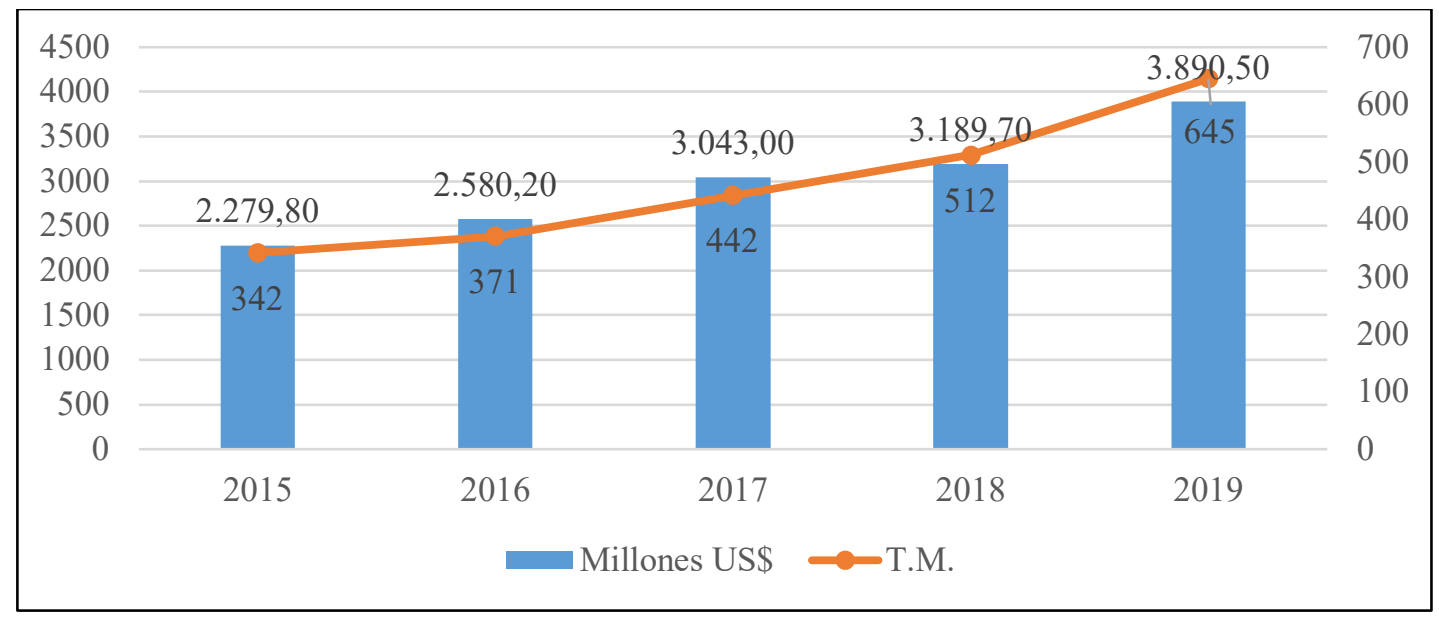

Fuente: Ministerio de Acuacultura y Pesca (2018). 
En lo relacionado a las exportaciones de camarón, según información de la Cámara Nacional de Acuacultura (CNA, 2020): en el año 2015 se produjeron 342 toneladas métricas de camarón con un total de 2.279,80 millones de dólares, con un crecimiento sostenido tanto en producción como en ventas que para el año 2019 alcanzaron la cifra récord de 3.890,50 millones de dólares con un total de 675 mil toneladas métricas, como se puede observar en el gráfico 1.

Datos proporcionados por el Ministerio de Acuacultura y Pesca (2018): los principales destinos del camarón ecuatoriano se encuentran en el mercado asiático, donde Vietnam representa el $38 \%$ del total de las exportaciones, seguido de China con el 16\%, a ellos les sigue Estados Unidos con el $16 \%$, el $7 \%$ España, Francia 6\% e Italia 6\%. En el gráfico 2 se exponen los destinos del camarón ecuatoriano.

Gráfico 2. Destino del camarón ecuatoriano.

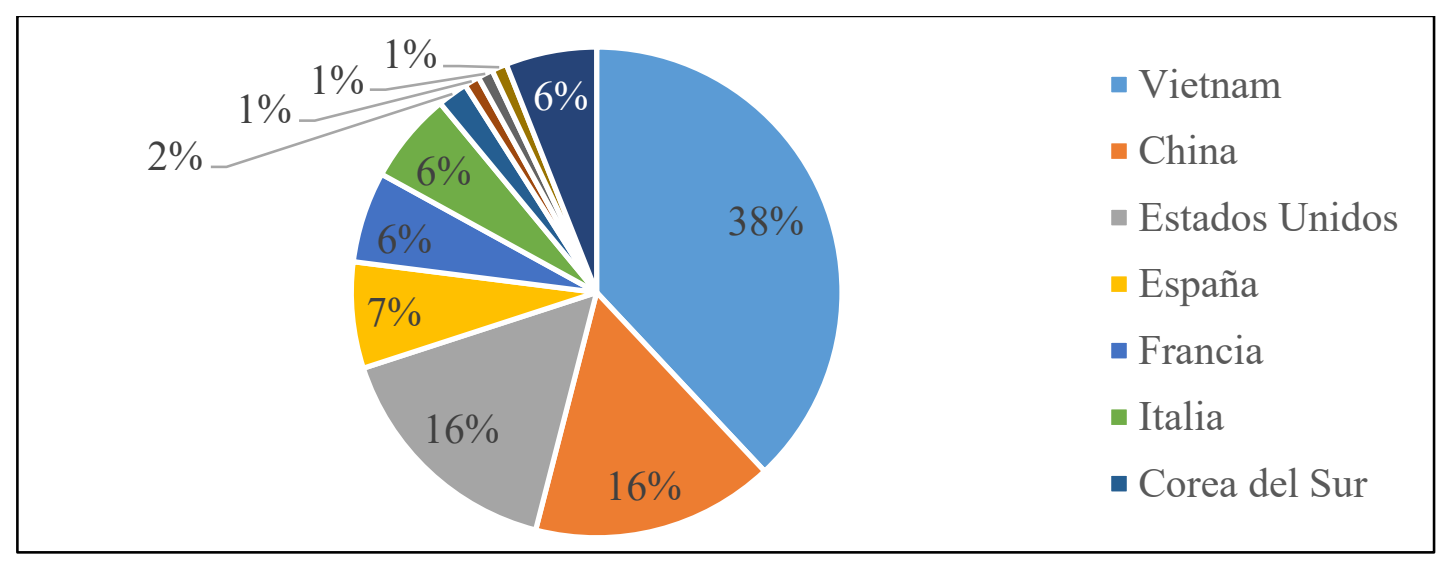

Fuente: Ministerio de Acuacultura y Pesca (2018).

Como indica Muñoz, Durán y González (2017): el sector camaronero es el segundo de mayor importancia para el país dentro del sector exportador no petrolero, por debajo del bananero, situación que debe ser apoyada por los gobiernos de turno para la implantación de estrategias integrales para competir con mejores condiciones en el mercado externo. En este sentido, Moncada- 
Sánchez, Ramírez-Quevedo y González-Illescas (2020): señalan que el crecimiento se lo ha logrado por mejoras de la cadena productiva, aplicación de mejores larvas con menor mortalidad, cambios sistemas productivos, insumos de calidad, mejor infraestructura lo que se ha visto reflejado en una mayor productividad para el fortalecimiento de las actividades camaroneras.

\subsection{Blockchain en el sector camaronero}

La actual industria camaronera ecuatoriana busca adoptar la tecnología blockchain. Esta idea se planteó al analizar la importancia de agregar valor a las exportaciones de camarón. El punto de partida es que las granjas camaroneras deben incluir certificación ambiental y social, lo que significa que no se utilizan antibióticos dentro de los procesos productivos, además del uso razonable de agua y estándares de trazabilidad en el proceso.

El camarón ecuatoriano ha logrado posicionarse como un producto con buena reputación en el mercado internacional por los estándares de calidad e higiene, y durante muchos años ha apostado por la sostenibilidad atrayendo consumidores responsables. Como exterioriza Enriquez (2020): los esfuerzos para aumentar la trazabilidad poniendo información a sus consumidores relacionada a sus orígenes de producción a través de la tecnología blockchain que ha sido integrada de manera reciente se ha convertido en un elemento diferenciador que ha provocado grandes expectativas de la industria para este nicho de la industria.

En conformidad con la Cámara Marítima del Ecuador (CAMAE, 2020a): la industria acuícola ecuatoriana se ha convertido en el primero a nivel mundial de agregar el blockchain a sus etapas productivas. El producto cuenta con código QR en los empaques para que el cliente y/o usuario tenga información sobre su trazabilidad la misma que se relaciona a las actividades productivas, su procedencia, alimentación, prácticas organizacionales y ambientales, empresa exportadora, garantizando de esta forma la transparencia y calidad 
del producto, exponiéndose la responsabilidad social corporativa utilizada por la organización.

La Cámara Marítima del Ecuador (CAMAE, 2020b): manifiesta que entre las empresas camaroneras que han implementado el blockchain dentro de sus operaciones se encuentran: Omarsa, S.A., Songa, C.A., Santa Priscila, S.A., Promarisco, S.A. Estas empresas utilizan el programa IBM Food Trust para una mejor trazabilidad.

Como enseña Porxas y Conejero (2018): la trazabilidad es uno de los principios básicos de la seguridad alimentaria, y es fundamental para poder comprender todo el historial del producto cuando se produce una alarma alimentaria, y así proteger la salud de los consumidores.

Por esta razón que la legislación europea estipula la obligación de definir un sistema de trazabilidad para asegurar que los alimentos puedan ser identificados y sus trazas puedan ser rastreadas en todas las fases de producción, transformación y distribución, asegurando un producto transparente y de calidad dentro del mercado europeo.

Para un mejor entendimiento se realiza un flujograma de las empresas que utilizan blockchain. En la figura 1, se muestra la información de forma transparente de la cadena logística mejorando la seguridad del producto.

Figura 1. Diagrama de flujo del almacenamiento de datos en blockchain.

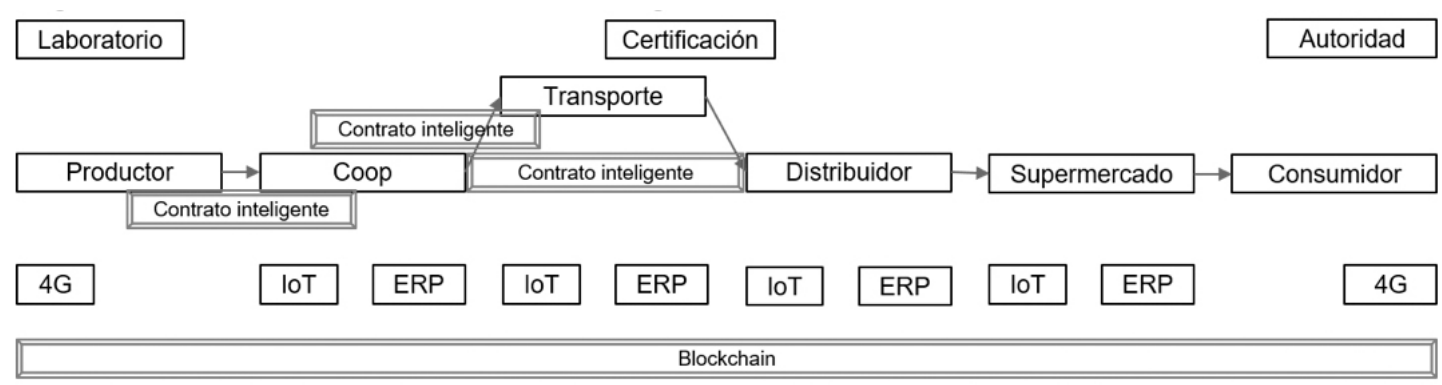

Fuente: Borrero (2019).

Una de las ventajas del blockchain con las bases de datos tradicionales 
que se manejan en las actividades comerciales son que estas últimas manejan datos centralizados que provocan baja confiabilidad porque provienen de una sola entidad, mientras que el blockchain es todo lo contrario, bloques que tienen acceso muchas personas para que la información pueda ser compartida, tal como lo indica la figura 2.

Figura 2. Diferencia entre blockchain y una base de datos tradicional.
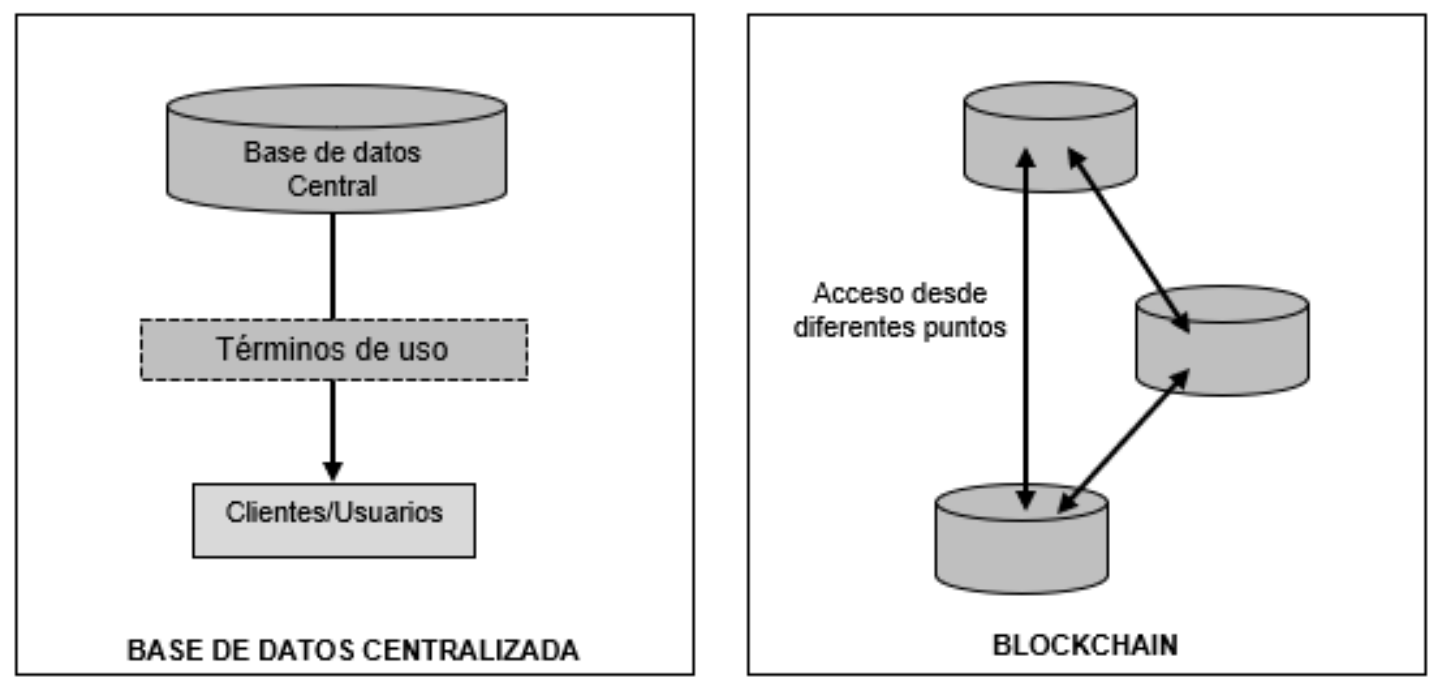

Fuente: Las Autoras (2021).

\section{Conclusiones}

La validez de los resultados se ajusta a la recopilación bibliográfica a través del método descriptivo y análisis documental que se convirtió en el ideal para este tipo estudio, donde la pandemia provocada por el COVID-19 limita la investigación de campo. Por esta razón se puede considerar que el blockchain se está convirtiendo en una alternativa para el sector camaronero ecuatoriano, donde la implementación de esta tecnología acredita al sector agrícola y acuícola dando a conocer el origen y destino del producto aminorando cualquier falencia o fraude durante el proceso logístico.

El blockchain cuenta con un potencial enorme para un control eficiente de su trazabilidad dentro de las empresas comerciales con gran transparencia 
para los usuarios que tengan acceso, a su vez indica que las organizaciones generarán mayor confiabilidad al contar con certificados que demuestren sus prácticas amigables con el medio ambiente.

Las empresas deben su éxito organizacional a la calidad de sus actividades productivas y comerciales, por lo que la responsabilidad social corporativa se convierte en una necesidad que asegure sus buenas prácticas laborales y ambientales, cumpliendo con su rol dentro de la sociedad. Para cumplir con la Responsabilidad Social Corporativa (RSC) se puede utilizar la tecnología blockchain para transparentar las actividades comerciales, donde el sector camaronero ecuatoriano se ha convertido en el pionero de implantarla, asegurando la calidad del producto al consumidor.

El camarón ecuatoriano se exporta a distintos destinos y continentes, donde la tecnología blockchain se puede convertir en un elemento para exponer la trazabilidad del producto desde su producción hasta llegar al consumidor, por parte de las empresas exportadoras de camarón de la provincia de El Oro, Ecuador, que a su vez puede servir como ejemplo para las compañías exportadoras de banano que es el otro sector dinamizador con que cuenta la provincia.

No cabe duda de que la aplicación de diferentes tecnologías en el sector acuícola ha incrementado la producción y comercialización, de esta manera el blockchain asegura que la calidad de los productos consumidos por los usuarios sea excelente, marcando el uso de tecnologías en este campo en un antes y después.

La transparencia de los procedimientos relacionados con la producción del crustáceo permite que los participantes tengan pleno acceso a la información pudiendo conocer el insumo utilizado para su alimentación, frecuencia, productor, en qué condiciones climáticas, laboratorio involucrado en proveer las larvas, y otro tipo de información diversa pero que son importantes para tener impacto positivo con el consumidor final. 
Las certificaciones se convierten en un mecanismo que confirman las buenas prácticas laborales y ambientales para elaborar un producto de calidad que cumpla con las especificaciones técnicas y demás requerimientos exigidos por los mercados internacionales para su ingreso con destino al consumidor final.

De esta forma, el blockchain se convierte en una aplicación tecnológica que genera ventaja competitiva al sector camaronero sobre las que no han integrado este tipo de tecnología, donde el mayor beneficiado será el consumidor.

\section{Referencias}

Benito, S. (2019). Presentación: Responsabilidad social corporativa en la industria alimentaria. Dossieres EsF, (35), 4-6, e-ISSN: 2603-848X. Recuperado de:

https://dialnet.unirioja.es/servlet/articulo?codigo $=7080422$

Borrero, J. (2019). Sistema de trazabilidad de la cadena de suministro agroalimentario para cooperativas de frutas y hortalizas basado en la tecnología Blockchain. CIRIEC-España: Revista de Economía Pública, Social y Cooperativa, (95), 71-94, e-ISSN: 1989-6816.

Recuperado de: https://doi.org/10.7203/CIRIEC-E.95.13123

CAMAE (2020a,b). Camarón ecuatoriano contará con tecnología Blockchain para garantizar la trazabilidad de su producción. Ecuador: Cámara Marítima del Ecuador.

Cavagnaro, J. (2018). Las 500 mayores empresas del Ecuador. Vistazo, (Especial), 140-154. Ecuador: Editorial Vistazo.

CNA (2020). Camarón - Reporte de Exportaciones Ecuatorianas Totales.

Ecuador: Cámara Nacional de Acuacultura. Recuperado de: https://www.cna-ecuador.com/estadisticas/

Enriquez, H. (2020). Tecnología blockchain en cadenas de suministro; 
mito, realidad o proyección. Revista de la Facultad de Ciencias Económicas, 24(1), 173-188, e-ISSN: 1668-6365. Recuperado de: https://doi.org/10.30972/rfce.2414366

Dávila-López, K., Carvajal-Romero, H., \& Vite-Cevallos, H. (2019). Análisis de rentabilidad económica del camarón (Litopenaeus vannamei) en el sitio Balao Chico, provincia del Guayas. Polo de Conocimiento, 5(1), 450-476, e-ISSN: 2550-682X. Recuperado de:

https://polodelconocimiento.com/ojs/index.php/es/article/view/1233

Esparza, N., Crespo, N., González, M., \& Fernández, A. (2020). Análisis de la solución blockchain aplicada a las cadenas de suministro en la ciudad de Babahoyo. Journal of Science and Research, 5(CININGEC), 831-844, e-ISSN: 2528-8083. Recuperado de:

https://doi.org/10.5281/zenodo.4451458

FAO (2018). El estado mundial de la pesca y la acuicultura: Cumplir los objetivos de desarrollo sostenible. ISBN: 978-92-5-130688-8. Roma: Organización de las Naciones Unidas para la Alimentación y la Agricultura.

Ganne, E. (2018). ¿Pueden las cadenas de bloques revolucionar el comercio internacional?. ISBN: 978-92-870-4765-6. Ginebra, Suiza: Organización Mundial del Comercio.

López, D. (2019). Blockchain: la revolución industrial de Internet. Ucudal: Revista de Derecho, 15(19), 197-201, e-ISSN: 2393-6193. Recuperado de: https://doi.org/10.22235/rd.v0i19.1721

Machado, F., Guayasamín, C., \& Montoya, P. (2020). Blockchain: Perspectiva y desafíos desde el comercio internacional. Revista Publicando, 7(23), 14-23, e-ISSN: 1390-9304. Recuperado de: https://revistapublicando.org/revista/index.php/crv/article/view/2057 Ministerio de Acuacultura y Pesca (2018). Reporte estadístico de exportación de camarón. Ecuador: Subsecretaría de Acuacultura. 
Recuperado de:

http://acuaculturaypesca.gob.ec/wp-content/uploads/2019/01/reporteestadistico-octubre-2018.pdf

Moncada-Sánchez, G., Ramírez-Quevedo, P., \& González-Illescas, M. (2020). Estrategias competitivas de las empresas ecuatorianas exportadoras de camarón. Casos de éxito. Innova Research Journal, 5(1), 111-128, e-ISSN: 2477-9024. Recuperado de:

https://doi.org/10.33890/innova.v5.n1.2020.1115

Muñoz, M., Durán, F., \& González, M. (2017). Análisis del sector camaronero ecuatoriano y sus ventajas competitivas y comparativas para encarar un mercado internacional competitivo. Conference Proceedings, 1(1), 1-8, ISSN: 2588-056X. Ecuador: Universidad Técnica de Machala.

Porxas, N., \& Conejero, M. (2018). Tecnología blockchain: funcionamiento, aplicaciones y retos jurídicos relacionados. Actualidad jurídica Uría Menéndez, (48), 24-36, e-ISSN: 1578-956X. Recuperado de: https://dialnet.unirioja.es/servlet/articulo?codigo $=6635980$

Varela-Véliz, H., Elizalde, B., Solórzano, S., \& Varela-Véliz, G. (2017). Exportación de camarón de la provincia de EI Oro en el contexto del Tratado Comercial con la Unión Europea. Espacios, 38(61), 1-19, e-ISSN: 0798-1015. Recuperado de:

https://www.revistaespacios.com/a17v38n61/17386124.html

Vega, F., Apolo, N., \& Sotomayor, J. (2019). La productividad del sector camaronero en la Provincia de el Oro y su impacto al medio ambiente. Agroecosistemas, 7(1), 39-44, e-ISSN: 2415-2862. Recuperado de: https://aes.ucf.edu.cu/index.php/aes/article/view/240 Zabala, V., \& Sánchez, J. (2019). Ecuador es el segundo exportador mundial de camarón. Ecuador: Grupo Ekos. 


\section{Sabi Samanta Martínez Prado}

e-mail: ssmartinez est@utmachala.edu.ec

Nacida en Machala, El Oro, Ecuador, el 1 de diciembre del

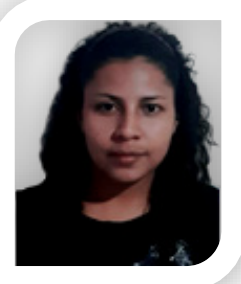
año 1990. Graduada en el Instituto Superior Tecnológico Ismael Pérez Pazmiño (INSTIPP), en la Especialidad de Comercialización y Ventas; soy Egresada de la Universidad Técnica de Machala (UTMACH), en la Carrera de Comercio Exterior; realización y aprobación del curso de inglés intermedio, curso de Computación Básica en la Universidad Técnica de Machala; Asesora en el proyecto denominado: Asesoría en importaciones a artesanos productores independientes o asociaciones de la Provincia de El Oro, Ecuador. 


\section{Verónica Karina Lascano Campoverde e-mail: vlascano est@utmachala.edu.ec}

Nacida en Machala, El Oro, Ecuador, el 2 de octubre del

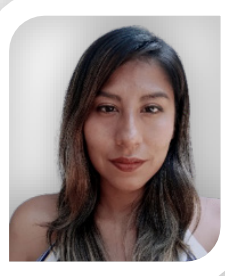
año 1989. Técnico en Comercio y Administración, graduada en el en el Instituto Superior Tecnológico Ismael Pérez Pazmiño (INSTIPP), en la Especialidad de Comercialización y Ventas; soy Egresada de la Universidad Técnica de Machala (UTMACH), en la Carrera de Comercio Exterior; realización y aprobación del curso de inglés intermedio, curso de Computación Básica en la Universidad Técnica de Machala; Asesora en el proyecto denominado: Asesoría en importaciones a artesanos productores independientes o asociaciones de la Provincia de El Oro, Ecuador; Participación en el proyecto de vinculación con la Asociación de Productores de Café Ecológico Lago Agrio (APROCEL): "Visión de exportación de café molido hacia el mercado internacional" realizado en Las Lajas, Ecuador. 


\section{Artículo Original / Original Article}

\section{Sandra Sayonara Solórzano Solórzano \\ e-mail:ssolorzano@utmachala.edu.ec}

Nacida en Machala, Ecuador, el 1 de septiembre del año

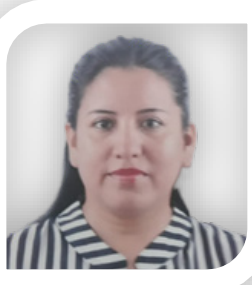
1975. Docente investigadora de la Universidad Técnica de Machala (UTMACH); Ingeniera en Comercio Internacional titulada en la Universidad Técnica de Machala; Magister en Administración de Empresas por la Universidad Tecnológica Empresarial de Guayaquil (UTEG); Coordinadora de la Carrera de Comercio Internacional en la Universidad Técnica de Machala; ocho años de experiencia en docencia universitaria; he publicado libros y artículos científicos; Pertenezco a grupos de Investigación; miembro de diferentes proyectos de Investigación.

El contenido de este manuscrito se difunde bajo una Licencia de Creative Commons ReconocimientoNoComercial-Compartirlgual 4.0 Internacional 